Crystal Struct ure Transf or mat i on and Di el ectric Properti es of Pol yner Composites I ncor por at i ng Zi nc Oxi de Nanor ods

\begin{tabular}{|l|l|}
\hline 著者 & $\begin{array}{l}\text { Yu Ji nhong, Wh Wei, Dai Dan, Song Yi ngze, Li } \\
\text { Chaoyang, Ji ang Nan }\end{array}$ \\
\hline $\begin{array}{l}\text { j our nal or } \\
\text { publ i cat i on t i t l e }\end{array}$ & Nacr ond ecul ar Resear ch \\
\hline vol une & 22 \\
\hline number & 1 \\
\hline page r ange & $19-25$ \\
\hline year & 2014 01- 31 \\
\hline URL & ht t p: //hdl . handl e. net /10173/1347 \\
\hline
\end{tabular}




\title{
Crystal Structure Transformation and Dielectric Properties of Polymer Composites Incorporating Zinc Oxide Nanorods
}

\author{
Jinhong $\mathrm{Yu}^{1}$, Wei $\mathrm{Wu}^{3}{ }^{*}$,Dan Dai ${ }^{1}$, Yingze Song ${ }^{1}$, Chaoyang $\mathrm{Li}^{4}$, and Nan Jiang*, \\ ${ }^{1}$ Ningbo Institute of Material Technology \& Engineering, Chinese Academy of Sciences, Ningbo, 315201, China ; \\ ${ }^{2}$ Guangxi Scientific Experiment Center of Mining, Metallurgy and Environment, Key Laboratory of New Processing \\ Technology for Nonferrous Metals and Materials, Ministry of Education, School of Material Science and \\ Engineering, Guilin University of Technology, Guilin 541004, China; \\ ${ }^{3}$ Department of Polymer Science and Engineering, Shanghai Jiao Tong University, Shanghai 200240, China \\ ${ }^{4}$ Institute for Nanotechnology, Kochi University of Technology, Kochi 782-8502, Japan \\ *Corresponding author, Electronic mail: yujinhong@glut.edu.cn (W. Wu)
}

\begin{abstract}
.
Zinc oxide ( $\mathrm{ZnO})$ nanorods were synthesized using a modified wet chemical method. Poly(vinylidene fluoride-co-hexafluoropropylene), P(VDF-HFP), nanocomposites with different $\mathrm{ZnO}$ nanorods loadings were prepared via a solution blend route. Field emission scanning electron microscopic (FESEM), X-ray diffraction (XRD), Fourier-transform infrared spectroscopy (FT-IR) were used to investigate the structure and morphology of the nanocomposites. XRD and FT-IR data indicate that the incorporation of $\mathrm{ZnO}$ nanorods promote the crystalline structure transformation of $\mathrm{P}(\mathrm{VDF}-\mathrm{HFP})$. As the content of $\mathrm{ZnO}$ nanorods increases, the $\beta$ phase structure increases while the $\alpha$ phase decreases. In addition, the dielectric properties of the P(VDF-HFP) and its composites were systematically studied.
\end{abstract}

Key words: ZnO nanorods, P(VDF-HFP), crystal structure transformation, dielectric properties, composites 


\section{Introduction}

In recent years poly(vinylidene fluoride) (PVDF) and its copolymers such as poly(vinylidene fluoride-chlorotrifluoroethylene) (P(VDF-CTFE)), Poly(vinylidene $\quad$ fluoride-co-hexafluoropropylene) (P(VDF-HFP)), have attracted considerable attention owing to their unique properties and widespread applications such as transducer, electrostriction actuator ${ }^{[1,2]}$. Specially, $\mathrm{P}(\mathrm{VDF}-\mathrm{HFP})$ exhibits high chemical resistance and high flexibility as a result of the presence of HFP. Additionally, it is more interesting that the introduction of HFP does not change the crystalline structure while mediating the degree ${ }^{[3,4]}$. In other words, the copolymer P(VDF-HFP) exhibits almost the same crystal structure as PVDF. It is well known that PVDF has four types of crystalline forms: $\alpha, \beta, \delta$ and $\gamma$. The chain conformation of $\alpha$ phase and $\beta$ phase are trans-gauche-trans-gauche' (TGTG') and tans-trans-trans-trans (TTTT) respectively. Through the $\beta$ phase is not a stable phase compared with the $\alpha$ phase, it still raises a lot of research interest, since it has an impressive effect on the piezoelectric and pyroelectric properties $^{[5]}$. To date, numerous fillers, such as nanoclay ${ }^{[6,7]}$, carbon nanotubes ${ }^{[8]}$, and graphene sheets ${ }^{[9,10]}$ have been used as an effective method for the forming of $\beta$ phase PVDF. However, there are only several literatures about P(VDF-HFP) based composites. For example, Giannelis et $\mathrm{al}^{[2]}$ studied a composite based on P(VDF-HFP) and clays and found that the introduction of organically modified clays promotes the transformation from $\alpha$ phase to $\beta$ phase. Randall et $\mathrm{al}^{[5]}$ investigated a composite made by $\mathrm{P}(\mathrm{VDF}-\mathrm{HFP})$ and kaolinite clay nanofillers, and their studies suggest that the incorporation of high aspect ratio fillers do not favor the formation of polar phase. All those studies indicate that the fillers play an indispensible role in the crystallization process of P(VDF-HFP). To the best of our knowledge, there has been no report detailing investigations on the effect of the one dimensional rod-like filler on the crystalline structures of P(VDF-HFP) copolymers.

In our study, zinc oxide $(\mathrm{ZnO})$ nanorods were employed as the active filler due to its unique properties. $\mathrm{ZnO}$ is a well-known multifunctional semiconductor material with a direct wide-band gap (3.37eV) and a large excition binding energy $(60 \mathrm{mV})^{[11]}$. Inspired by the enormous applications in actuators, high performance sensors, transducers and nanogenerators, $\mathrm{ZnO}$ have been studied since the $1960 \mathrm{~s}{ }^{[12]}$. The properties of $\mathrm{ZnO}$ are different froms the bulk when it comes to nanoscale, which is due to the quantum confinement of charge carriers within the nanostructures. What's more, the shape of the $\mathrm{ZnO}$ crystal has a great effect on the electronic properties ${ }^{[13]}$. Recently, Wang et al studied the dielectric properties of PVDF based nanocomposite by introducing ZnO nanowire custers and $\mathrm{ZnO}$ nanowire bundles and the incorporation of $\mathrm{ZnO}$ exhibits a good contribution to the enhancement 
of dielectric constant in their researches ${ }^{[14,15]}$.

Herein, a well-defined $\mathrm{ZnO}$ nanorods was successively synthesized via a modified wet chemical route and the corresponding hybrid nanocomposites made of P(VDF-HFP) and ZnO nanorods (NRs) have been fabricated using a solution blend method. The morphology and microstructure of the composites were investigated by a combination of field emission scanning electron microscope (FESEM), wide-angle X-ray scattering (WAXS), Fourier-transform infrared spectroscopy (FTIR). The dielectric properties of the samples were investigated with dielectric relaxation spectroscopy (DRS).

\section{Experimental section}

\subsection{Materials}

Zinc acetate dihydrate $\left(\mathrm{C}_{4} \mathrm{H}_{6} \mathrm{O}_{4} \mathrm{Zn} \cdot 2 \mathrm{H}_{2} \mathrm{O}\right)$, N,N-dimethyform amide (DMF), ethanol, acetone and sodium hydroxide were all analytical grade and purchased from Sinopharm Chemical Reagent co., Ltd, China. The P(VDF-HFP)(SOLEF 11008/0003) pellet was provided by Solvay Shanghai Co. China. All the chemicals were used as-received without any further treatment.

\subsection{Synthesis of $\mathrm{ZnO}$ nanorods}

$\mathrm{ZnO}$ nanorods were prepared according to a modified method formerly reported by Host Weller et al ${ }^{[16-18]}$ In brief, $21.95 \mathrm{~g}(0.1 \mathrm{~mol})$ of zinc acetate dehydrate and $120 \mathrm{ml}$ ethanol were placed in a flask equipped with a mechanical stirrer and thermometer. Firstly, the mixture was stirred at $70{ }^{\circ} \mathrm{C}$ for 30 minutes, then a solution of $7.2 \mathrm{~g}$ ( $0.18 \mathrm{~mol})$ sodium hydroxide in $60 \mathrm{ml}$ ethanol was added drop-wisely within $5 \mathrm{~min}$. The resulting mixture was subsequently heated for three days at $80{ }^{\circ} \mathrm{C}$ while vigorously agitating. During this time, a certain amount of deionized water was injected. After the reactions, white crystalline products were harvested by centrifugation and thorough washings with deionized water and ethanol.

\subsection{Preparation of P(VDF-HFP)/ZnO nanorod nanocomposites}

$\mathrm{P}(\mathrm{VDF}-\mathrm{HFP}) / \mathrm{ZnO}$ nanorod nanocomposites were prepared through a solution blend method. An appropriate amount of $\mathrm{P}(\mathrm{VDF}-\mathrm{HFP})$ was added to the $\mathrm{ZnO}$ nanorods suspension and stirred vigorously overnight at room temperature. The mixture was then poured into a methanol bath and vigorously stirred for 5-10 min. The resulting precipitates were filtered and dried under vacuum oven at $80{ }^{\circ} \mathrm{C}$ for $24 \mathrm{~h}$. The dried samples were then hot-pressed into rectangular die at $180{ }^{\circ} \mathrm{C}$. For comparison between the pristine and the filled polymer samples, the neat $\mathrm{P}(\mathrm{VDF}-\mathrm{HFP})$ sample was prepared with the same process.

\subsection{Characterization}

A field emission scanning electron microscope (SEM, JEOL JEM-7401, Tokyo, Japan) were used to study the 
morphology of both the as-synthesis $\mathrm{ZnO}$ nanorods and the nanocomposites. For measurement, a proper amount of $\mathrm{ZnO}$ nanorods were dispersed in ethanol and then dropped onto the surface of Si wafer slides. The nanocomposites specimens were broken and the fractured surface was sputtered with thin layers of gold to avoid the accumulation of charge. Fourier-transform infrared spectroscopy (FT-IR) was conducted with a Perkin-Elmer Paragon 1000 instrument over the range of 4000-650 $\mathrm{cm}^{-1}$.Wide-angle X-ray Diffraction (XRD) patterns were recorded on a Rigaku D/MAX-2200/PC automatic diffractometer (Rigaku Corporation, Tokyo, Japan), all measurements were performed at the atmospheric pressure and room temperature with nickel-filtered $\mathrm{Cu}$ target Ka radiation at $40 \mathrm{KV}$ and $20 \mathrm{~mA}$ with a scintillation counter system. Data were recorded in a range of $2 \theta=20-80^{\circ}$ at a scanning rate of $5 \%$ min and a range of $2 \theta=2-30^{\circ}$ at a scanning rate of $2 \% \mathrm{~min}$ for $\mathrm{ZnO}$ nanorods and composites samples respectively. Specially, for the nanocomposites, the data collected by scanning the top surface of the film samples. Dielectric properties were measured using an impedance analyzer (Aglient 4294A) with 16451B Dielectric Test Fixture in the frequency range of $100 \mathrm{~Hz}-10 \mathrm{MHz}$. For temperature dependence of the dielectric properties, the measurements were performed from $20^{\circ} \mathrm{C}$ to $140^{\circ} \mathrm{C}$.

\section{Results and discussion}

\subsection{Characterization of zinc oxide nanorods}

Figure 1 shows a SEM image of the synthesized ZnO nanorods. The nanorods display high regularity with a calculated length varying from $120 \mathrm{~nm}$ to $250 \mathrm{~nm}$ and a diameter varying from $20 \mathrm{~nm}$ to $30 \mathrm{~nm}$. The aspect ratio of the as-prepared nanorods lies in the range of about 10 . Wide angle X-ray diffraction measurement was conducted to check the crystallity of our products. Figure 2 shows the XRD pattern of as-synthesis $\mathrm{ZnO}$ nanorods. Obviously, all the diffraction peaks can be indexed to the Wurtzite-structured (hexagonal) ZnO (JCPDSNo.36-1451) and no other phases can be detected.

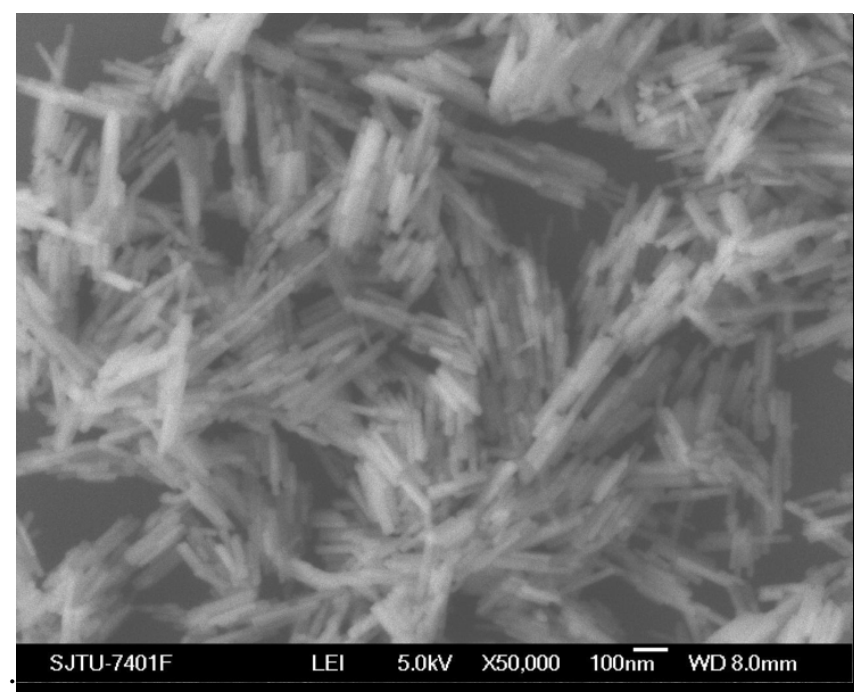


Figure 1. SEM overview image of ZnO nanorods morphology

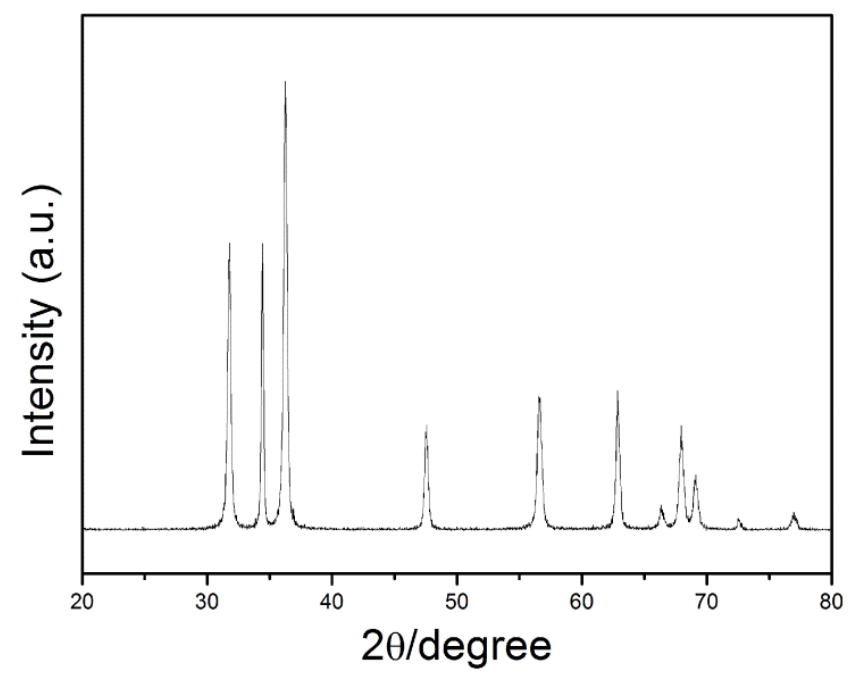

Figure 2. XRD patterns of the as-synthesis $\mathrm{ZnO}$ nanorods

\subsection{Morphology of P(VDF-HFP) and its nanocomposites}
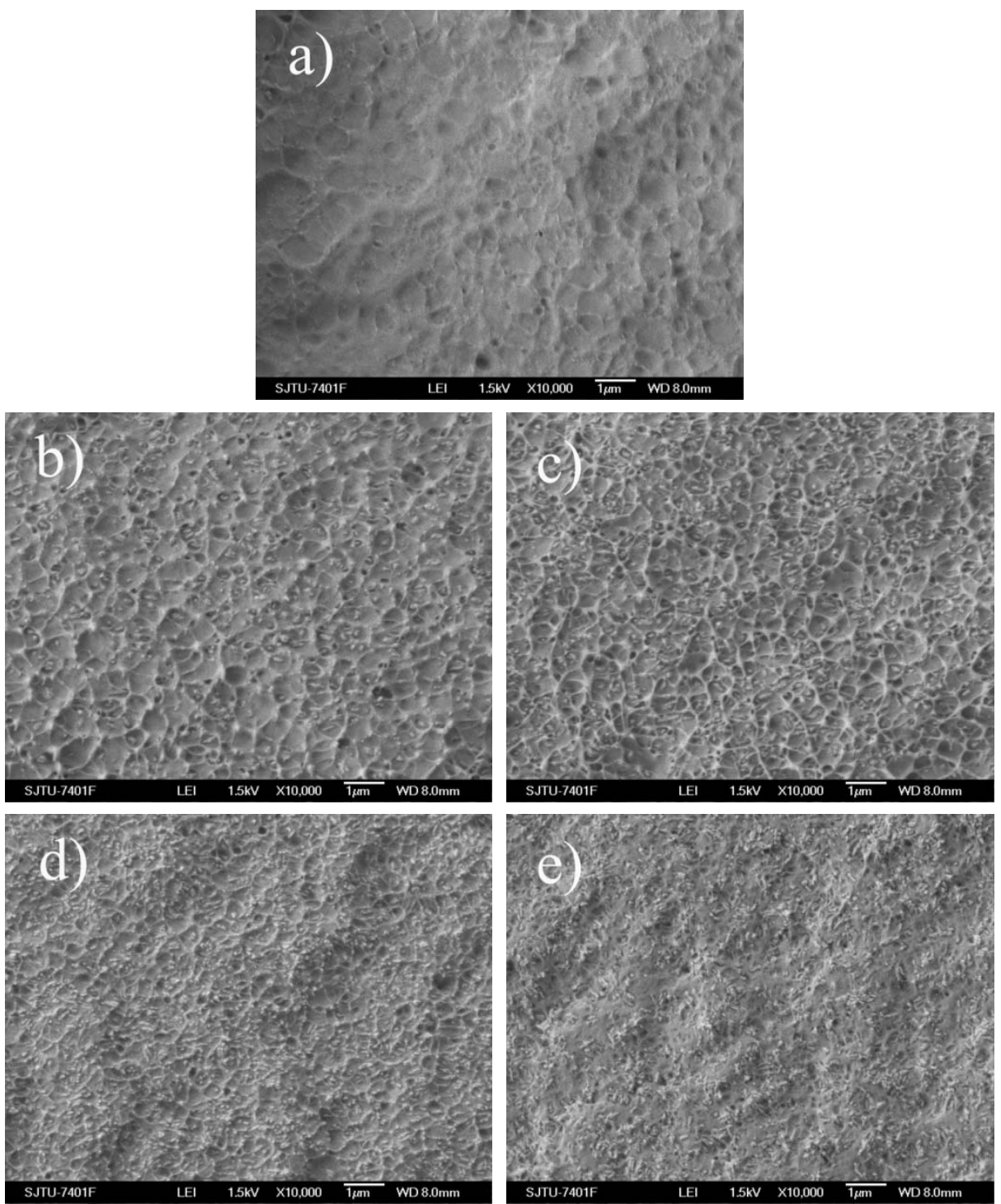

Figure 3. SEM microstructure of P(VDF-HFP) nanocomposites with different ZnO nanorods loadings : a) 0wt\%, 


\section{b) $10 w t \%, c) 20 w t \%, d) 30 w t \%, e) 40 w t \%$}

The quality of $\mathrm{ZnO}$ nanorods dispersion in the polymer matrix directly correlated with its properties, such as dielectric properties. A comparison of the SEM images of the cross section of the neat P(VDF-HFP) and its nanocomposite are obtained in Figure 3. In the case of the neat P(VDF-HFP), as shown in Figure 3(a), the fractured morphology shows the typical fracture way of polyolefin. Figure 3(b-d) shows fractured morphology of the nanocomposites with weight fraction of $\mathrm{ZnO}$ nanorods at 10, 20, 30, and $40 \mathrm{wt} \%$. It is obvious to see from four images that most $\mathrm{ZnO}$ nanorods are uniformly dispersed within P(VDF-HFP) matrix and are fractally agglomerated only at high filler loading from the fractured cross-surfaces of the nanocomposites. Meanwhile, the fracture morphology of the nanocomposites display similar to that of the neat P(VDF-HFP), suggesting that the existence of ZnO nanorods did not significantly alter the fracture mechanism of the matrix.

\subsection{Crystal structure transformation of P(VDF-HFP) and its nanocomposites}

P(VDF-HFP) copolymers, unlike P(VDF-TrFE) and P(VDF-TFE) copolymers, exhibit similar crystal structure as homopolymer PVDF. It is reasonable to study the patterns of P(VDF-HFP) in reference to the standard patterns of PVDF ${ }^{[3,5]}$. Figure 4 displays the XRD patterns of both neat $\mathrm{P}(\mathrm{VDF}-\mathrm{HFP})$ and $\mathrm{P}(\mathrm{VDF}-\mathrm{HFP})$ nanocomposites. It is clearly seen that there are four peaks which correspond to the $\alpha(100), \alpha(020), \alpha(110)$, and $\alpha(021)$ crystal plane [5]. A slight difference in the intensity of each peaks is observed between the neat P(VDF-HFP) and the nanocomposite with $10 \mathrm{wt} \% \mathrm{ZnO}$ nanorods. When the content of $\mathrm{ZnO}$ increases further, the nanocomposites display not only a reduced peak intensity but also disappearance of some peaks and occurrence of a new reflection peak. It is well known that the incorporation of nanoparticles would result in the formation of $\beta$ phase ${ }^{[6,19]}$. In our study, it was found that when the content of $\mathrm{ZnO}$ nanorods reach as high as $20 \mathrm{wt} \%$, the new developed $\beta$ phase can be easily seen, which is consistent with the study reported previously ${ }^{[2,7,20]}$. The decreased peak intensity of $\alpha$ phase crystalline planes is supposed to be due to the internal stress along the nanorod axis direction during the growth process. For the sake of the large specific surface area of $\mathrm{ZnO}$ nanorods, those nanorods tend to self-agglomerate into clusters in the matrix, particularly at a higher content. Consequently, the clusters might have lower potential to serve as "seeds" to initiate the growth of polymers crystal, and might also suppress the motion of the chain around them. Infrared spectroscopy measurement was conducted to give a further study of the crystal structure change of the hybrid nanocomposites. Figure 5 exhibits the FT-IR spectra of P(VDF-HFP) and its nanocomposites. The five absorption curves are almost of the same shape, which means the introduction of $\mathrm{ZnO}$ nanorods does not change the absorption behavior of P(VDF-HFP) heavily. The absorption peaks at $762.1 \mathrm{~cm}^{-1}$ and $795.8 \mathrm{~cm}^{-1}$ can be ascribed to the $\alpha$ phase and the absorption peak at $840.5 \mathrm{~cm}^{-1}$ is belongs to the $\beta$ phase ${ }^{[2,5]}$. And 
only when the content of $\mathrm{ZnO}$ nanorods reaches as high as $20 \mathrm{wt} \%$ or further, the absorption at $840.5 \mathrm{~cm}^{-1}$ shows up, which is in consistent with results of XRD measurement.

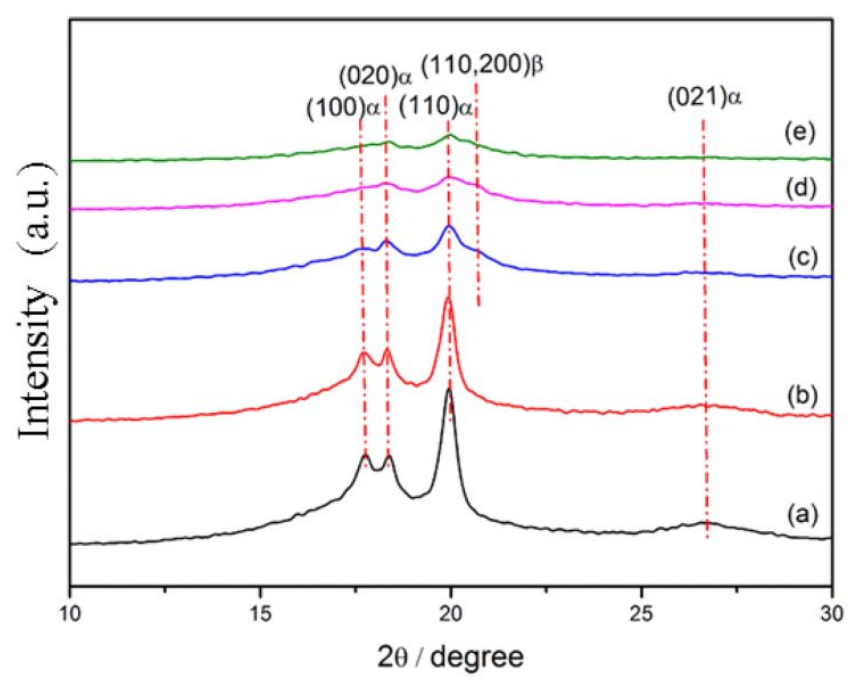

Figure 4. XRD patterns of P(VDF-HFP) and its nanocomposites : (a) 0wt \%, (b)10wt $\%$, (c)20wt $\%$, (d)30wt\%, (e) $40 \mathrm{wt} \%$

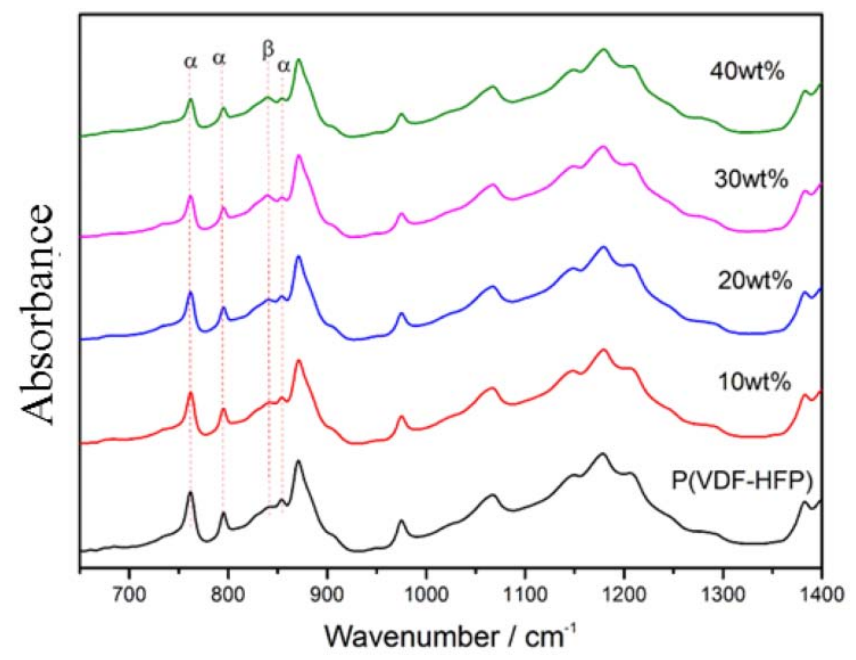

Figure 5. FTIR spectra of P(VDF-HFP) and its nanocomposites. The characteristics frequencies for $\alpha$ and $\beta$ phases are shown in the spectra.

\subsection{Glass transition temperature of P(VDF-HFP) and its nanocomposites}

The incorporation of fillers into a polymer matrix can bring about changes in the thermal characteristics of the resulting composites. One benchmark used to compare the thermal behavior of composites is the glass transition temperature (Tg). Figure 6 shows the change in Tg for the neat P(VDF-HFP) and its nanocomposites. It is seen that the neat $\mathrm{P}(\mathrm{VDF}-\mathrm{HFP})$ displayed a glass transition temperature at ca. $-36.2{ }^{\circ} \mathrm{C}$. Meanwhile, the Tgs of the nanocomposites with $10,20,30$, and $40 \mathrm{wt} \%$ are $-28.8,-24.5,-20.6$, and $20.1{ }^{\circ} \mathrm{C}$, respectively. With increasing the content of $\mathrm{ZnO}$ nanorods into the $\mathrm{P}(\mathrm{VDF}-\mathrm{HFP})$ matrix, Tgs significantly shifts to higher temperature. This phenomenon could be explained by the following aspects of factors. The increase in $\mathrm{Tg}$ with addition $\mathrm{ZnO}$ 
nanorods indicates that the mobility of the $\mathrm{P}(\mathrm{VDF}-\mathrm{HFP})$ molecule chains has been reduced after introducing $\mathrm{ZnO}$ nanorods into the matrix. It is believed that $\mathrm{ZnO}$ nanorods can act as physical interlock points in the polymer matrix, which generally restrains the molecular chain mobility.

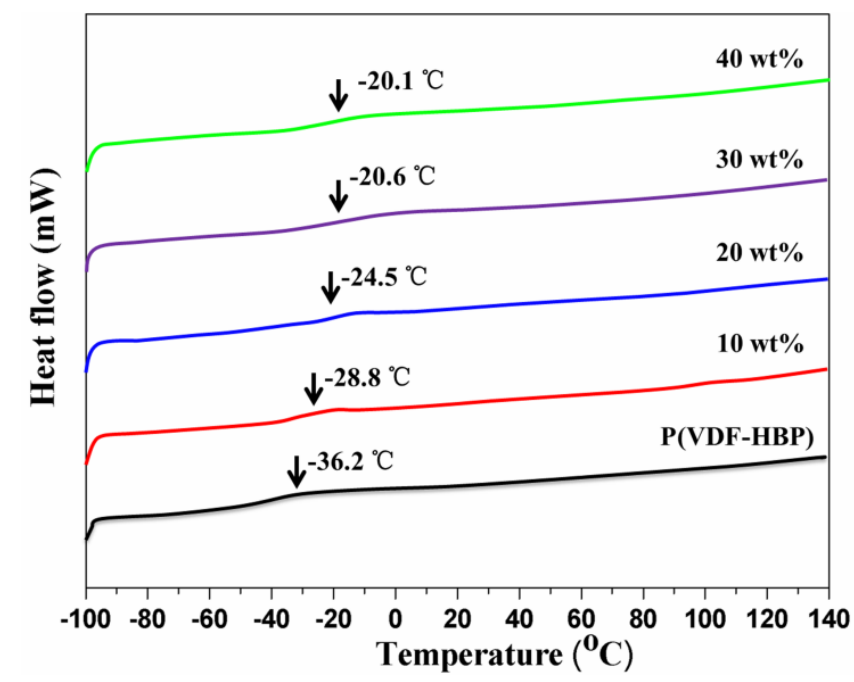

Figure 6. DSC curves of of P(VDF-HFP) and its nanocomposites.

\subsection{Dielectric properties of $\mathrm{P}(\mathrm{VDF}-\mathrm{HFP})$ and its nanocomposites}

The frequency dependence of dielectric constant and conductivity of the neat P(VDF-HFP) and its nanocomposites with different $\mathrm{ZnO}$ nanorods loadings were measured at room temperature (Figure 7 and Figure 8). Generally, the dielectric permittivity shows a monotonically increase with the increasing fillings and decreases as the frequency increases in our studies. We propose that the enhancement of the dielectric constant in the composites is resulted from two aspects. The incorporation of $\mathrm{ZnO}$ nanorods would induce large interfacial areas in composites which may result in a higher degree of interfacial polarization. On the other hand, the polarization and dielectric properties of the PVDF based ferroelectric polymers originate from their crystalline domain and the $\beta$ phase exhibits piezoelectric and pyroelectric properties ${ }^{[21-25]}$, so it is reasonable to deduce that the formation of $\beta$ phase do contribute to the enhancement of dielectric constant. And the decrease rate of the dielectric constant here is not remarkable. This can be related to the semi-conductive characteristic of ZnO. As illustrated in Figure 8, the conductivity of nanocomposites is only $10^{-7} \mathrm{~S} \mathrm{~m}^{-1}$ at $10^{3} \mathrm{~Hz}$ at room temperature, indicating that an excellent insulation performance is still maintained even though the loading is as high as $40 \mathrm{wt} \%$.

It is well known that the dielectric loss comes from three sources: the movement of molecular dipoles (dipolar loss), direct current (dc) conduction (transport-related loss) and space charge (interfacial polarization contribution). The dielectric loss of nanocomposites with varying weight fractions of $\mathrm{ZnO}$ nanorods are displayed in Figure 9. It is noteworthy that the dielectric loss of the $\mathrm{P}(\mathrm{VDF}-\mathrm{HFP}) / \mathrm{ZnO}$ nanrods nanocomposites is still no more than 0.275 
over the frequency range from $10^{3} \mathrm{~Hz}$ to $10^{7} \mathrm{~Hz}$. It is easy to see that the dielectric loss of the composites exhibits a completely different behavior at different frequency range. The dielectric loss increases at low frequencies and decreases in the high frequency range with the weight fraction of $\mathrm{ZnO}$ nanorods. It is widely accepted that the low-frequency process is mainly associated with the interfacial polarization and conductivity, whereas at higher frequencies, the dipolar relaxation process dominant. We suppose that the conduction loss might be attributed mainly to the conduction loss at low frequencies and this phenomenon will be discussed further in the following section. The decrease trend of the dielectric loss observed at high frequency is supposed to be resulted from the less contribution of dipoles relaxation, which is consistent with other reports ${ }^{[26]}$.

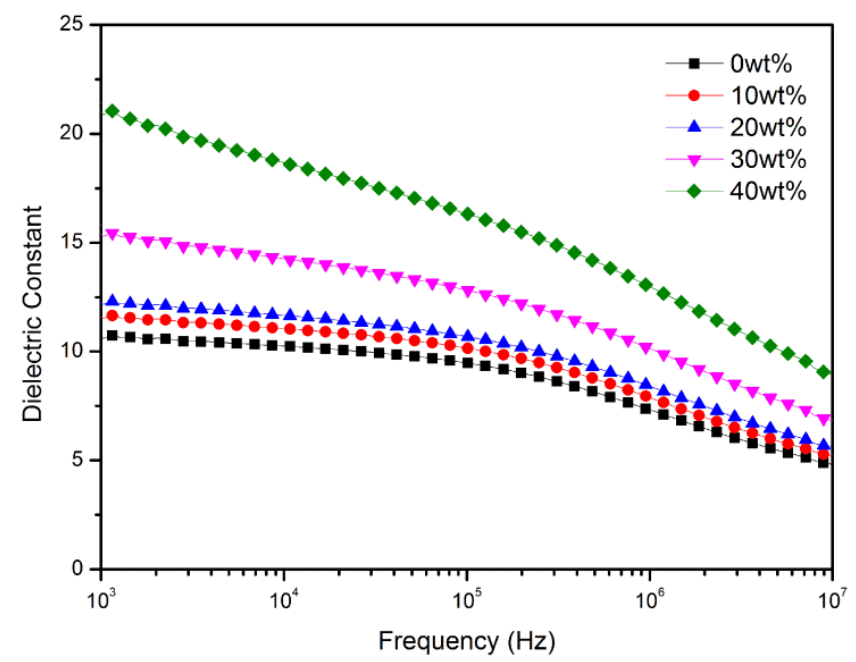

Figure 7. Frequency dependence of dielectric constant of pristine P(VDF-HFP) and its nanocomposites with different loading : 0wt\%,10wt\%,20wt\%,30wt\%,40wt\%.

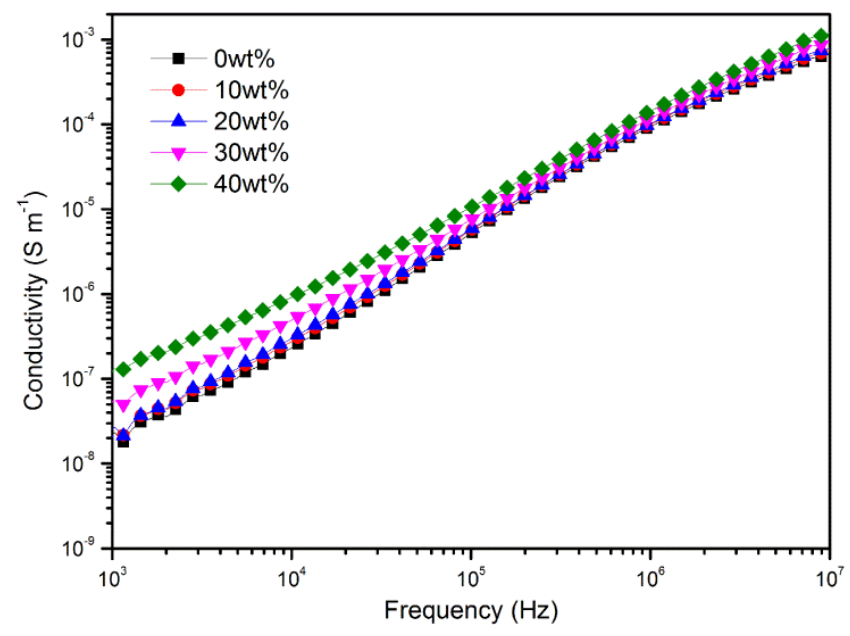

Figure 8. Frequency dependence of conductivity of pristine $\mathrm{P}(\mathrm{VDF}-\mathrm{HFP})$ and its nanocomposites with different loading : 0wt $\%, 10 \mathrm{wt} \%, 20 \mathrm{wt} \%, 30 \mathrm{wt} \%, 40 \mathrm{wt} \%$. 


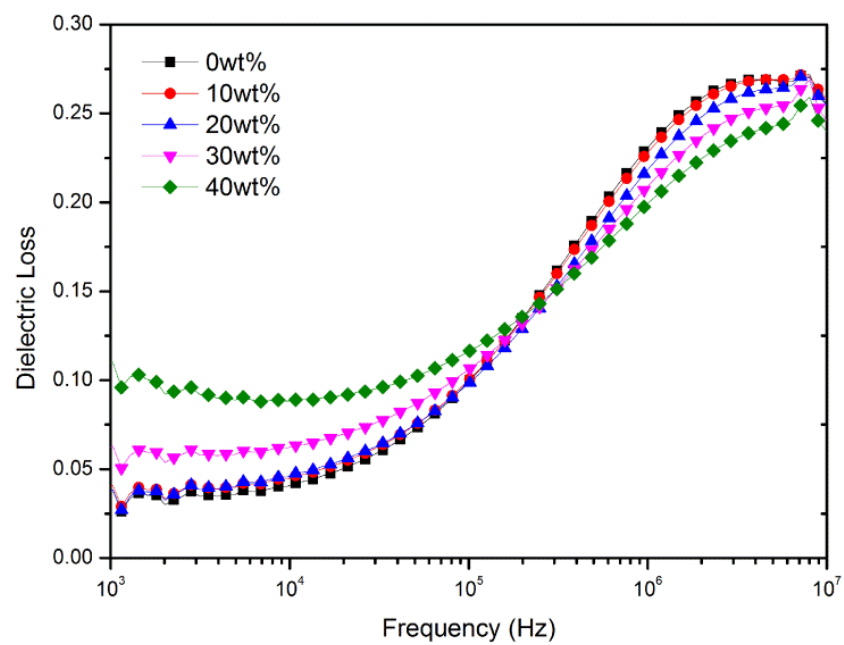

Figure 9. Frequency dependence of dielectric loss of pristine P(VDF-HFP) and its nanocomposites with different loading : 0wt\%,10wt $\%, 20 w t \%, 30 w t \%, 40 w t \%$.

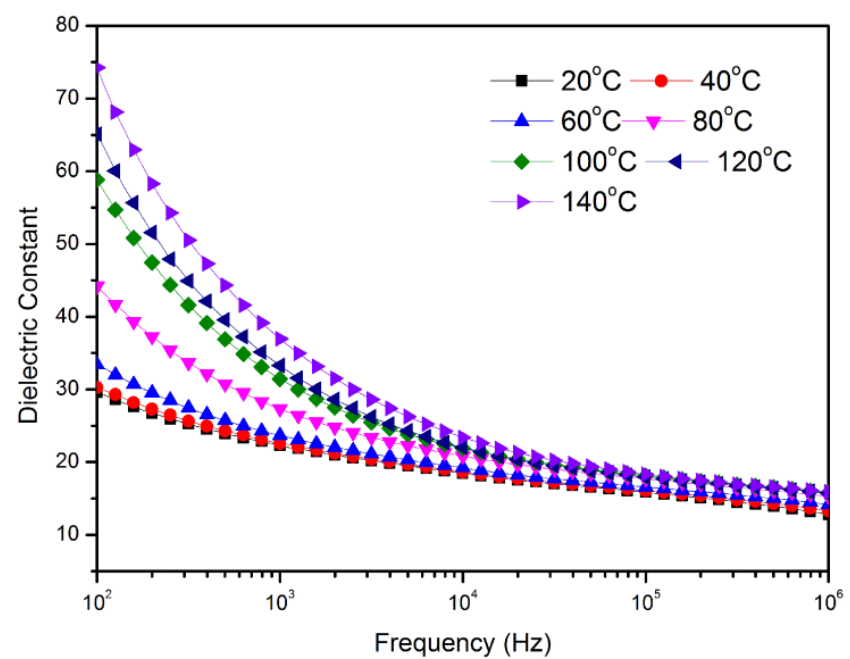

Figure 10. Temperature dependency of the dielectric constant of P(VDF-HFP)/ZnO nanorods (40wt\%)

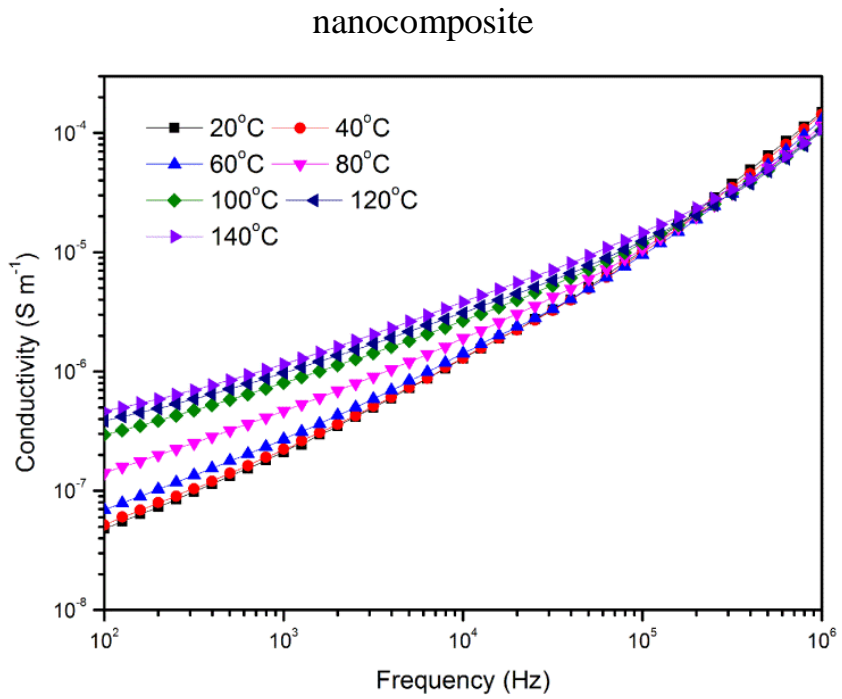

Figure 11. Temperature dependency of the conductivity of P(VDF-HFP)/ZnO nanorods (40wt\%)

nanocomposite 


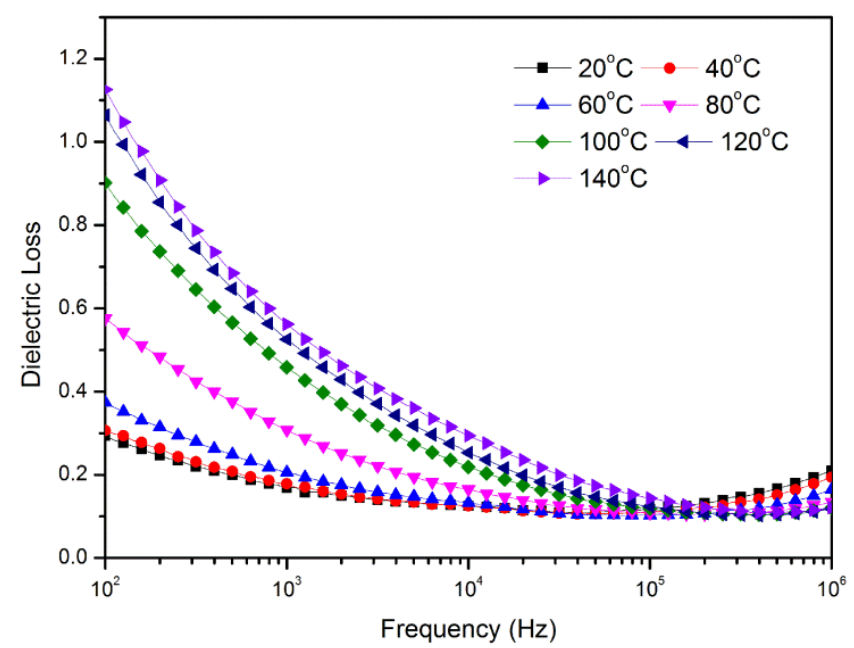

Figure 12. Temperature dependency of the dielectric loss of $\mathrm{P}(\mathrm{VDF}-\mathrm{HFP}) / \mathrm{ZnO}$ nanorods (40wt\%)

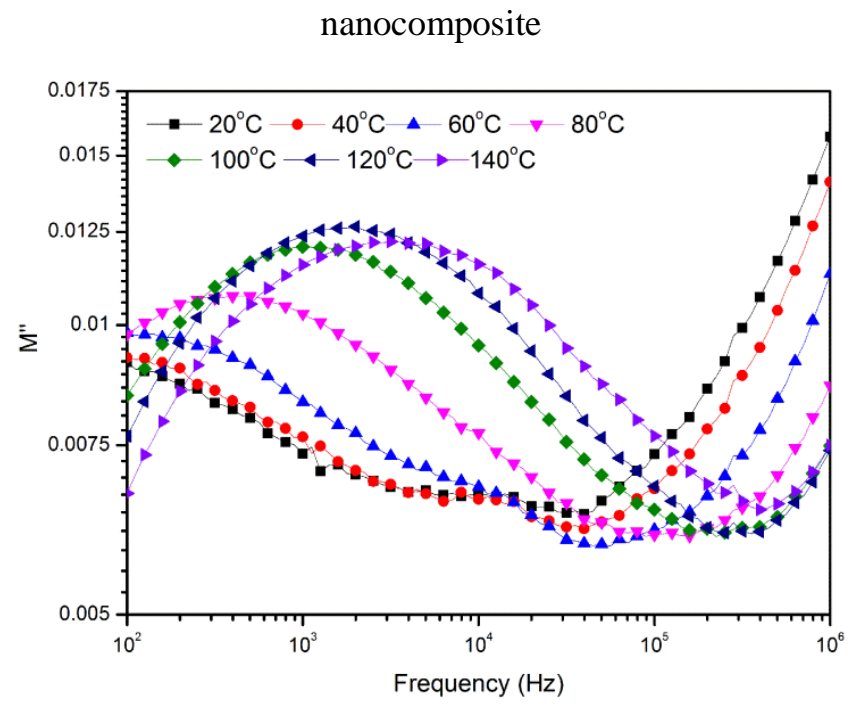

Figure 13. Temperature dependence of the imaginary part of electric modulus of P(VDF-HFP)/ZnO nanorods (40wt\%) nanocomposite

It is of great help to confirm the mechanism of the dielectric behavior to conduct temperature-dependent dielectric properties measurement. Figure 10 and Figure 11 present the temperature dependence of the dielectric constant and conductivity of $\mathrm{P}(\mathrm{VDF}-\mathrm{HFP}) / \mathrm{ZnO}$ nanorods (40wt\%) nanocomposite respectively. It is obvious that the temperature exerts an impressive effect on the dielectric properties of the composites (e.g., the dielectric constant of the composite at $140{ }^{\circ} \mathrm{C}$ and $100 \mathrm{~Hz}$ is as big as 74.26 which is about 2.5 times of the one at $20^{\circ} \mathrm{C}$ and $100 \mathrm{~Hz}$ ). The permittivity of the composite exhibits an increase trend over the full frequency range as the temperature increases especially at the low frequencies. This can be rationalized in terms of the influence of the increase in the conductivity of $\mathrm{ZnO}$ nanorods which can be seen in Figure $\mathbf{1 1}$ and the thermal expansion of the $\mathrm{P}(\mathrm{VDF}-\mathrm{HFP})$ as the temperature increases. A similar result was also reported for radial ZnO/PVDF composites ${ }^{[14]}$. The dielectric loss also shows an increasing trend at low frequencies and decreases at high frequencies which can 
be seen from Figure 12. To determine the underlying physical mechanism of the relaxation behaviors, it is feasible to choose electric modulus (defined according to Equation (1)) to analyze our experimental data since it is independent of electrode nature and contact, space charge injection and absorbed impurity conduction ${ }^{[27,28]}$.

$$
M^{*}=\frac{1}{\varepsilon^{*}}=\frac{1}{\varepsilon^{\prime}-j \varepsilon^{\prime \prime}}=\frac{\varepsilon^{\prime}}{\varepsilon^{\prime 2}+\varepsilon^{\prime \prime 2}}+j \frac{\varepsilon^{\prime \prime}}{\varepsilon^{\prime 2}+\varepsilon^{\prime 2}}=M^{\prime}+j M^{\prime \prime}
$$

As shown in Figure 13, a temperature dependence of the imaginary part of electric modulus of the composite was established. The loss peak, which is related to the interfacial polarization loss, shifts towards higher frequencies with increasing temperature. It is reasonable to deduce that there would be a loss peak at frequency lower than $10^{2} \mathrm{~Hz}$. In other words, there is little interfacial polarization in all composites at room temperature when the frequency is above $10^{3} \mathrm{~Hz}$. All these results are also agreement with the discussion made above.

\section{Conclusions}

$\mathrm{P}(\mathrm{VDF}-\mathrm{HFP})$ based nanocomposites filled with different amount of $\mathrm{ZnO}$ nanorods were prepared by a solution blend method. It was found that the $\mathrm{ZnO}$ nanorods were well dispersed in the matrix. XRD, FTIR and DSC data indicate that the incorporation of $\mathrm{ZnO}$ nanorods render some change of the crystalline structure of P(VDF-HFP). As the content of $\mathrm{ZnO}$ nanorods increases, the $\beta$ phase structure can be obtained while the $\alpha$ phase decreases.

The dielectric properties of the composites with different $\mathrm{ZnO}$ nanorods contents were studied. The dielectric permittivity exhibits a monotonically increase with the increasing $\mathrm{ZnO}$ nanorods content. The temperature dependence of the dielectric properties of the composite with a loading of $40 \mathrm{wt} \% \mathrm{ZnO}$ nanorods was investigated. And it was found that the dielectric constant of the composite at $140{ }^{\circ} \mathrm{C}$ and $100 \mathrm{~Hz}$ is as big as 74.26 which is about 2.5 times of the one at $20^{\circ} \mathrm{C}$ and $100 \mathrm{~Hz}$.

\section{Acknowledgments}

Guangxi Small Highland Innovation Team of Talents in Colleges and Universities, and Guangxi Funds for Specially-appointed Expert.

\section{References}

[1] V. K. Tiwari, P. K. Kulriya, D. K. Avasthi, P. Maiti, J. Phys. Chem. B 113, 11632-11641 (2009).

[2] A. Kelarakis, S. Hayrapetyan, S. Ansari, J. Fang, L. Estevez, E. P. Giannelis, Polymer, 51, 469-474 (2010).

[3] F. Guan, J. Pan, J. Wang, Q. Wang, L. Zhu, Macromolecules, 43, 384-392 (2010).

[4] F. Guan, J. Wang, J. Pan, Q. Wang, L. Zhu, Macromolecules, 43, 6739-6748 (2010).

[5] V. Tomer, E. Manias, C. A. Randall, J. Appl. Phys, 110, 044107 (2011).

[6] D. Shah, P. Maiti, D.Jiang, C. A. Batt, E. P. Giannelis, Adv. Mater., 17, 525-528 (2005). 
[7] L. Priya, J. P. Jog, J. Polym. Sci. Part B: Polym. Phys, 40, 1682-1689 (2002).

[8] S. Manna, A. K. Nandi, J. Phys. Chem. C, 111, 14670-14680 (2007).

[9] J. Yu, P. Jiang, C. Wu, L. Wang, X. Wu, Polym. Compos., 32, 1483-1491(2011).

[10] S. Ansari, E. P. Giannelis, J. Polym. Sci. Part B: Polym. Phys., 47, 888-897 (2009).

[11] Z. Wang, Mater. Sci. Engineer.: R: Reports, 64, 33-71 (2009).

[12] P. Samanta, S. Basak, P.R. Chaudhuri, Mater. Today, 14, 295 (2011).

[13] S. Polarz, Adv. Funct. Mater., 21, 3214-3230 (2011).

[14] G. Wang, Y. Deng, Y. Xiang, L. Guo, Adv. Funct. Mater., 18, 2584-2592 (2008).

[15] G. Wang, Y. Deng, L. Guo, Chem. Eur. J., 16, 10220-10225 (2010).

[16] H. Oliver, C. Pacholski, H. Weller, A. Yasuda, J. M.Wessels, Nano Lett., 3, 1097-1101 (2003).

[17] P. Claudia, K. Andreas, H. Weller, Angew. Chem. Int. Ed., 41, 1188-1191 ( 2002).

[18] M. Voigt, M. K. Nzer, H. Thiem, W. Peukert, J. Phys. Chem. C, 114, 6243-9 (2010).

[19] D. Shah, P. Maiti, E. Gunn, F. Schmidt, D. Jiang, C. A. Batt, E. P. Giannelis, Adv. Mater., 16, 1173-1176 (2004).

[20] P. Thomas, S. Satapathy, K. Dwarakanath, K. B. R. Varma. eXPRESS Polym. Lett., 4, 632-643 (2010).

[21] N. Levi, R. Czerw, S. Xing, P. Iyer, D. L. Carroll, Nano Lett., 4, 1267-1271 (2004).

[22] G. H. Kim, S.M. Hong, Y. Seo, Phys. Chem. Chem. Phys., 11, 10506-10512 (2009).

[23] S. P. Bao, G. D. Liang, S. C. Tjong, Carbon, 49, 1758-1768 (2011).

[24] J. K.Yuan, Z. M. Dang, S. H.Yao, J.W. Zha, T. Zhou, S. T. Li, J. Bai, J. Mater. Chem., 20, 2441 (2010)

[25] J. Li, J. Claude, L. E. Norena-Franco, S. I. Seok, Q. Wang, Chem. Mater., 20, 6304 - 6306 (2008).

[26] W. Yang, S.Yu, R. Sun, R. Du, Acta Mater., 59, 5593-5602 (2011).

[27] C. V. Chanmal, J.P. Jog, eXPRESS Polym. Lett., 2, 294-301 (2008).

[28] G. Ioannou, A. Patsidis, G. C. Psarras, Composites Part A, 42, 104-110 (2011). 